\author{
Amir Pirouzian ${ }^{\star}$ \\ Johns Hopkins University, Wilmer Eye Institute, 600 \\ N. Wolfe Street, Baltimore, MD 20027, USA \\ Dates: Received: 01 September, 2015; Accepted: \\ 27 November, 2015; Published: 30 November, 2015 \\ *Corresponding author: Amir Pirouzian, M.D. \\ Wilmer Eye Institute, Johns Hopkins University \\ School of Medicine, 600 North Wolfe Street \\ Baltimore, MD 21287, USA, Tel: 858-248-0747; \\ E-mail: apirouzian@gmail.com; apirouz2@jhmi.edu \\ www.peertechz.com \\ Keywords: Cornea; Scar; Child; Trauma; \\ Transplantation; Femtosecond laser; Keratoplasty
}

\author{
Case Report
}

\section{Femtosecond Assisted Deep Lamellar Keratoplasty in a 41/2 Year Old Child for Traumatic and Infectious Corneal scar- A Case Report}

\section{Introduction}

Femtosecond laser technology was originally introduced into the clinical ophthalmology market to create all-inclusive laser-assisted refractive platforms for cornea-flap construction in the early 2000s. US FDA subsequently approved femtosecond laser technology for laser-enabled keratoplasty in mid 2000s [1]. The unique feature of the femtosecond laser is in its distinctive capacity to generate a variety of customized trephination patterns in both donor and recipienthost corneas in keratoplasty procedures [1,2]. The customized shapes which have been investigated in the literature are "top hat", "Christmas tree", "mushroom", "zigzag" and "hexagonal". Each of these customized shapes has a number of particular advantages in a specific clinical setting. As an example, the "top hat" shape is recommended in diseases involving corneal endothelium and the "mushroom" trephination pattern is recommended for corneal surface diseases [3-5]. Femtosecond laser platforms which are currently available for laser-enabled keratoplasty (FSLEK) are Intralase (AMO, Irvine, CA), FEMTEC (Bausch \& Lomb, Rochester, NY) and Technolas ${ }^{\mathrm{TM}}(20 / 10$ Perfect Vision, Heidelberg, Germany). The newer generations of femtosecond lasers have increasingly higher energy pulses and allow for shorter tissue ablation period and faster cut times.

In this article, we describe, using for the first time, the zigzag shape FS-assisted deep lamellar keratoplasty using the $4^{\text {th }}$ generation
Intralase ${ }^{\mathrm{TM}}$ in a $41 / 2$ year old child with a deep central stromal scar. The major advantage of this technique is to allow for an earlier suture removal, which can possibly reduce the higher graft-rejection rate seen in children following penetrating keratoplasty. As a result, faster visual rehabilitation can also be initiated.

\section{Case Presentation}

A $4 \frac{1}{2}$ year-old male with a 10 -month old history of nonperforating right corneal injury and subsequently resolved Aspergillus corneal infection due to trauma was referred to our clinic. His bestcorrected distance visual acuity for the right eye was 2 LogMAR (Counting figures) at presentation. His pupils were normal and no afferent pupillary defect was present. Anterior segment examination showed a centrally irregular anterior to deep stromal scar of $4 \mathrm{~mm}$ in size of moderate opacity distorting visual axis (Figure 1). No corneal vascularization was present. The anterior chamber was deep and quite. The iris, lens, vitreous and retina were normal. Intraocular pressures were also normal. The optic nerves were normal and no cupping was present. The best-corrected distance visual acuity of the left eye was 0 LogMAR. The patient had been undergoing occlusion therapy 3-4hrs/day on the left eye for the right eye amblyopia treatment during the past 4-months. Right eye anterior segment optical coherence tomography, ASOCT (Visante', Carl Zeis Meditec, Dublin, CA) and ATLAS (Carl Zeis Meditec, Dublin, CA) corneal 
topography were performed (Figures 2a,3). AS-OCT showed the depth of corneal opacity to be at $410 \mathrm{um}$. Central corneal thickness was measured to be at $560 \mathrm{um}$. Irregular mires and astigmatism was present on the corneal topography. Patient had also been intolerant of contact lens wear. After reviewing available treatment options, parents were carefully counseled on risks/benefits/alternatives and potential complications of FALK and consented to the procedure.

\section{Surgery}

Institutional review board of University of California, Irvine, granted one-time approval to perform this procedure following an in-depth risk/benefit analysis, ethical assessment and providing the necessary guideline to maintain optimal safety profile throughout the procedure. Informed consent adhering to the Helsinki protocols was

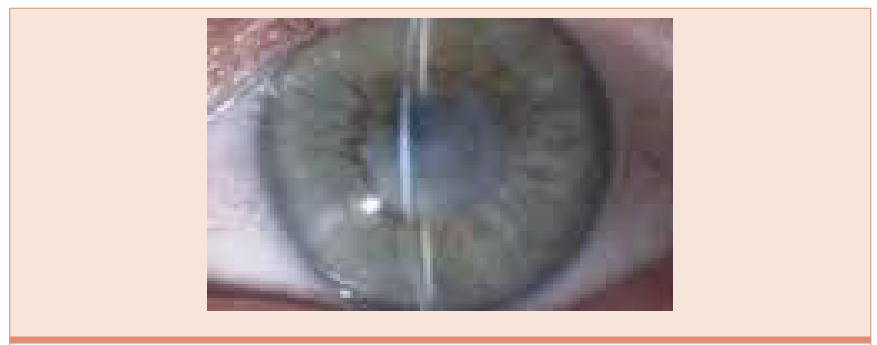

Figure 1: Pre-operative photograph showing the central scar.

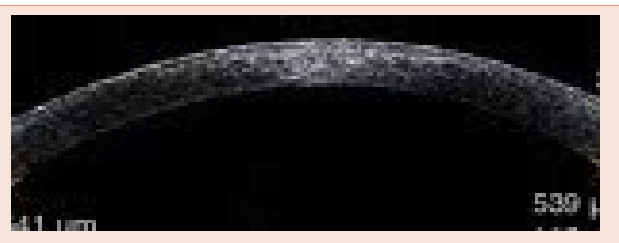

Figure 2a: Anterior segment optical coherence tomography of the right eye.

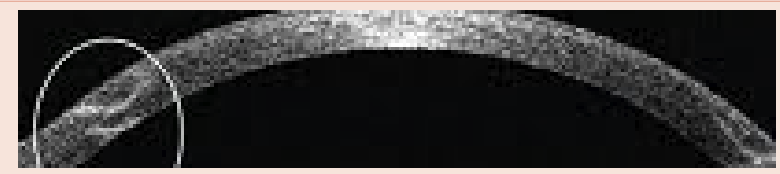

Figure 2b: Anterior segment optical coherence of a post-keratoplasty zigzag wound.

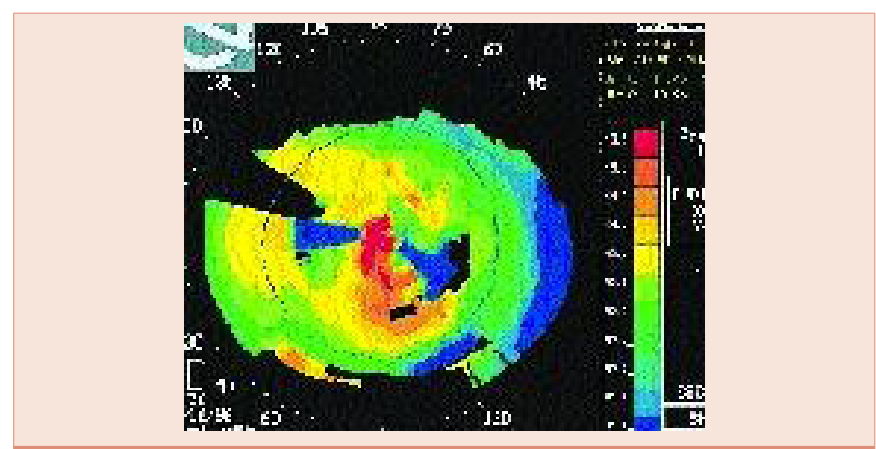

Figure 3: ATLAS Corneal topography of the right eye showing distorted central mires. obtained from parents following a thorough discussion related the off-label FDA of performing FS-assisted keratoplasty in children, and to the risks, benefits and alternatives involved with this procedure including general anesthesia, femtosecond platform and transport from the laser room to the adjacent surgical room. Patient underwent general anesthesia and a flexible J-tube was used for intubation at the University of California, Irvine and medical center. The induction was done in the holding area. The tube was securely taped against the lower jaw to avoid any contact with the FS-laser machine. Pediatric anesthesia team had rolled the anesthesia-air support machine into the laser room. Using the depth of cornea scar measured at 410um, the posterior lamellar cut was set at $470 \mathrm{um}$. Eyelid speculum was not used. Separation of eyelids was achieved manually using the right and left index fingers of an assistant. Following the docking of the suction ring onto the applanation cone under the laser, a brief episode of acute bradycardia (30beats/min) occurred as a result of oculocardiac reflex. A $0.5 \mathrm{cc}$ of $0.5 \mathrm{mg}$ atropine sulfate was intravenously administered to restore the normal heart rate and the laser procedure was continued without any further incidence.

The 4-th generation Intralase ${ }^{\mathrm{TM}}$ laser (Abbott Medical Optics, Santa Ana, CA) was used to create a deep lamellar cut on the right eye of the patient. Patient was transported from the adjoining laser room to the operating room while intubated and the right eye was prepped in the usual manner. A previously cut donor cornea graft using the femtosecond laser with zigzag pattern from Sight Life ${ }^{\mathrm{TM}}$ Eye Bank (Florida, USA) was available. The settings are shown in (Tables 1,2). Using the "big bubble" air-technique, detachment of descemet's membrane was achieved. The surgery was carried out as described in the literature. 3 Interrupted sutures were placed at $50 \%$ depth of the host and graft cornea junction at the pre-marked locations (Figure 2b). Surgery was concluded without complication.

\section{Post-operative Course}

In day one of the post-operative visit, patient's graft cornea was clear and the junction between the graft and host cornea was sealed. The uncorrected visual acuity was at 1.0 LogMAR and the pinhole visual acuity was 0.7 LogMAR (Figure 4). No epithelial defect was present. Anterior chamber had no cells with mild flare. The patient was placed on prednisolone acetate $1 \%$ (Pred Forte, Alcon Inc. Ft Worth, TX) hourly and Moxifloxacin 0.5\% (Vigamox, ALCON, Ft. Worth, TX) four times a day. Lubrication was also prescribed hourly. Father was instructed to see the original referring cornea ophthalmologist within three days. It was reported that four sutures were removed after two months by the referring ophthalmologist. Six additional sutures were removed at four months and the final two sutures were removed at six months. Patient was tapered to Loteamx (Loteprednol etabonate $0.5 \%$, Bauch and Lomb Inc., Rochestor, NY) once daily and the final refractive error at six months was reported to be $-1.50+2.50 \times 33$ OD with visual acuity of $0.4 \log M A R$. The patient continued occlusion therapy for amblyopia treatment. Graft remained clear at six months post-operatively.

\section{Discussion}

A femtosecond laser assisted deep lamellar keratoplasty (FALK) provides multitude of advantages over conventional penetrating 


\begin{tabular}{|l|l|}
\hline \multicolumn{2}{|l|}{ Table 1: Femtosecond (Intralase) zig-zag B-parameters for the host cornea. } \\
\hline Femtosecond laser frequency & $60 \mathrm{KHZ}$ \\
\hline Anterior cut depth & 270 \\
\hline Posterior side cut diameter & 8.6 \\
\hline Posterior cut depth & 470 \\
\hline Posterior side cut spot separation & 4 \\
\hline Posterior side cut angle & 30 \\
\hline Ring lamellar cut depth & 300 \\
\hline Ring lamellar out/inner diameter & $8.6 / 3.0$ \\
\hline Lamellar tangential/raida spot separation & $6.0 / 6.0$ \\
\hline Anterior side cut angle & 30 \\
\hline Anterior side cut spot/layer separation & $04-\mathrm{Apr}$ \\
\hline Alignment incisions & 12 \\
\hline
\end{tabular}

Table 2: Femtosecond (Intralase) zig-zag B-parameters for the donor cornea \{SightLife $®$, Florida, US\}.

\begin{tabular}{|l|l|}
\hline Femtosecond laser frequency & $60 \mathrm{KHZ}$ \\
\hline Posterior side anterior depth & 270 \\
\hline Posterior side cut diameter & 8.6 \\
\hline Posterior cut depth & 850 \\
\hline Posterior side cut spot/layer separation & $04-\mathrm{Apr}$ \\
\hline Posterior side cut angle & 30 \\
\hline Ring lamellar cut depth & 300 \\
\hline Ring lamellar outer/inner diameter & $8.6 / 7.4$ \\
\hline Lamellar tangential/raidal spot separation & $5.0 / 5.0$ \\
\hline Lamellar posterior depth & 330 \\
\hline Anterior diameter & 8.5 \\
\hline Anterior side cut spot/layer separation & $04-\mathrm{Apr}$ \\
\hline Alignment incisions & 12 \\
\hline
\end{tabular}

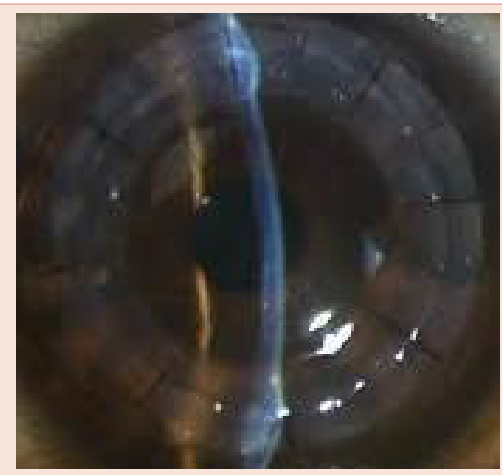

Figure 4: Post-operative photography day \#1.

and/or manually dissected deep lamellar keratoplasty in children. Femtosecond laser assisted zigzag trephination keratoplasty, in particular, has been shown to create a relatively precise donor-host cornea fit with an increased ease for graft suturing and reduction in inducing higher order aberration. This in turn would have a positive impact on the contrast sensitivity and quality of vision [5-11]. Due to the fact that the host's corneal endothelium is not disturbed, the risk of graft rejection decreases in comparison to conventional penetrating keratoplasty. Smoother corneal interfaces are made using the femtosecond lasers in comparison to conventional and manually dissected DALK [8]. Utmost effort should be taken, however, to match the depth of the suture placement in the donor and host cornea at $50 \%$ so as to achieve a true "lock and key" configuration. Improved sealing of the incision site permits the surgeon to use only enough suture tension to keep the incision apposed, reducing distortion from tight or multiple sutures. The increased surface area of these junction areas leads to improved tensile strength of the wound, allowing for earlier sutures removal and potentially reducing graft rejection [12]. Then patients can also be safely switched to less potent topical steroid medications which are known to have fewer long-term side effects for chronic maintenance of immune-suppressive therapy [13].

Here we safely performed and demonstrated the application of zigzag shaped femtosecond assisted laser deep lamellar keratoplasty in a young child with deep cornea scar. The general advantages of this technique in comparison to conventional manual DALK include 1. Allowing for an earlier suture removal, 2. Reduction of astigmatism in early stages of recovery, 3. For creation of smoother interface bed between the host and graft cornea, 4. Potential reduction in graft-rejection rate and 5. Improving the chances for faster visual rehabilitation.

The disadvantages, however, include requiring to 1 . Have the femtosecond laser platform in the operating room theater or at a very nearby location where the transport of an already intubated patient to the operating room may not impose or add further safety risk issues 2. Have general anesthesia and their essential equipment must be available in the laser room 3. Be watchful of potential oculo-cardiac reflex event at the time of laser ducking on the globe.

There have been three other published articles which have reported the use of femtosecond assisted laser keratoplasty in children with femtosecond laser assisted lamellar keratoplasty (mushroom shape or zigzag) for variety of other ocular diseases such as Avelino cornea dystrophy, keratoconus, and in congenital hereditary stromal dystrophy with a novel decorin gene mutation in a Korean family in the adult and penetrating keratoplasty in the child [14-17].

\section{Conclusion}

FS-enabled DALK holds numerous potential advantages over full thickness PK in children. As the globe is never penetrated during the surgery, intra-operative risks of expulsive hemorrhage are lowered. Reduced risk of rejection allows for earlier cessation and tapering of topical steroids or switching to less potent topical steroids which have higher safety profiles. The shaped trephination pattern of femtosecond laser-assisted lamellar keratoplasty allows for better wound integrity and apposition, less astigmatic induction, smoother interface bed between the host and donor bed allowing for potentially earlier visual recovery, thus decreasing the risk of progressive amblyopia and permanent loss of vision in children.

\section{References}

1. Ignacio TS, Nguyen TB, Chuck RS, Kurtz RM, Sarayba MA (2006) Top-hat wound configuration for penetrating keratoplasty using the femtosecond laser: a laboratory model Cornea 25: 336-340.

2. Steinert RF, Ignacio TS, Sarayba MA (2007) Top-hat shaped penetrating keratoplasty using the femtosecond laser. Am J Ophthalmol 143: 689-691.

3. Farid M, Kim M, Steinert RF (2007) Results of penetrating keratoplasty performed with a femtosecond laser zigzag incision: initial report. Ophthalmology 114: 2208-2212. 
4. Buratto L, Böhm E (2007) The use of the femtosecond laser in penetrating keratoplasty. Am J Ophthalmol 143: 737-742.

5. Price FW, Price MO (2008) Femtosecond laser shaped penetrating keratoplasty: one-year results utilizing a top-hat configuration. Am J Ophthalmol 145: 210-214.

6. Cheng YY, Tahzib NG, van Rij G, van Cleynenbreugel H, Pels E, et al. (2008) Femtosecond laser-assisted inverted mushroom keratoplasty Cornea 27 679-685.

7. Bahar I, Kaiserman I, Lange AP, Levinger E, Sansanayudh W, et al. (2009) Femtosecond laser versus manual dissection for top-hat penetrating keratoplasty. Br J Ophthalmol 93: 73-78.

8. Farid M, Steinert RF, Gaster RN, Chamberlain W, Lin A (2009) Comparison of penetrating keratoplasty performed with a femtosecond laser zig-zag incision versus conventional blade trephination. Ophthalmology116: 1638-1643.

9. Price FW Jr, Price MO, Jordan CS (2008) Safety of incomplete incision patterns in femtosecond laser-assisted penetrating keratoplasty. J Cataract Refract Surg 34: 2099-2103.

10. Chamberlain WD, Rush SW, Mathers WD, Cabezas M, Fraunfelder FW (2011) Comparison of femtosecond laser-assisted keratoplasty versus conventional penetrating keratoplasty. Ophthalmology 118: 486-491.

11. Chamberlain W, Omid N, Lin A, Farid M, Gaster RN, et al. (2012) Comparison of corneal surface higher-order aberrations after endothelial keratoplasty, femtosecond laser-assisted keratoplasty, and conventional penetrating keratoplasty. Cornea 31: 6-13.

12. Price FW Jr, Price MO, Grandin JC, Kwon R (2009) Deep anterior lamellar keratoplasty with femtosecond-laser zigzag incisions. J Cataract Refract Surg 35: 804-808.

13. Pirouzian A, Craven ER (2014) Critical appraisal of loteprednol ointment, gel, and suspension in the treatment of postoperative inflammation and pain following ocular and corneal transplant surgery. Clin Ophthalmol 8: 379-387.

14. Agarwal A, Brubaker JW, Mamalis N, Kumar DA, Jacob S, et al. (2009) Femtosecond-assisted lamellar keratoplasty in atypical Avellino corneal dystrophy of Indian origin. 6 year old child. Eye Contact Lens 35: 272-274

15. Buzzonetti L, Petrocelli G, Laorante A (2010) Anterior lamellar keratoplasty assisted by intralase femtosecond laser in a pediatric patient. J Pediatr Ophthalomol Strabismus 21: 47.

16. Buzzonetti L, Petrocelli G, Valente P (2012) Big-bubble deep anterior lamellar keratoplasty assisted by femtosecond laser in children. Cornea. 31:1083-6.

17. Kim JH, Ko JH, Lee I, Kim JY, Tchah H (2011) A novel mutation of the decorin gene identified in a Korean family with congenital hereditary stromal dystrophy. Cornea 30: 1473-1477.

Copyright: (c) 2015 Pirouzian A. This is an open-access article distributed under the terms of the Creative Commons Attribution License, which permits unrestricted use, distribution, and reproduction in any medium, provided the original author and source are credited. 